\title{
Decentralized Observers for Optimal Stabilization of Large Class of Nonlinear Interconnected Systems
}

\author{
Ghazi Bel Haj Frej \\ Centre de Recherche en Automatique de Nancy \\ CRAN-CNRS UMR 7039 \\ University of Lorraine, FRANCE \\ Research Unit MACS, ENIG \\ University of Gabes, Tunisia \\ Mohamed Boutayeb \\ Centre de Recherche en Automatique de Nancy \\ CRAN-CNRS UMR 7039 \\ University of Lorraine, FRANCE
}

\author{
Assem Thabet \\ Laboratoire de Recherche MACS, ENIG \\ University of Gabes, Tunisia \\ Mohamed Aoun \\ Laboratoire de Recherche MACS, ENIG \\ University of Gabes, Tunisia
}

\begin{abstract}
This paper focuses on the design of decentralized state observers based on optimal guaranteed cost control for a class of systems which are composed of linear subsystems coupled by nonlinear time-varying interconnections. One of the main contributions lies in the use of the differential mean value theorem (DMVT) to simplify the design of estimation and control matrices gains. This has the advantage of introducing a general condition on the nonlinear time-varying interconnections functions. To ensure asymptotic stability, sufficient conditions expressed in terms of linear matrix inequalities (LMIs) are established to compute the control and the observation gains of the overall system. High performances are shown through numerical simulation of a power system with three interconnected machines.
\end{abstract}

\section{General Terms}

Large scale interconnected system, Decentralized control

\section{Keywords}

Large Scale System, Interconnected System, Decentralized Observer, Feedback Control

\section{INTRODUCTION}

The problem of designing robust state observers/control for largescale systems has received considerable attention over the past few decades. The literature on this subject is very extensive, and includes a number of comprehensive surveys (see [19], [22] and the references there in). Indeed, large scale interconnected systems can be found in different fields as power systems, space structures, manufacturing processes, transportation networks, communication and others [15]. Designing a centralized state observers/controller for these systems may not be efficient due to the complexity/size of systems and the large number of operations to be performed in the real-time implementation [14]. These limitations motivate the design of decentralized control schemes.

In many practical situations, complete state measurements are not available at each individual subsystem for decentralized control. Consequently, one has to consider decentralized feedback control based on measurements only or design decentralized observers to estimate the state of individual subsystems that can be used for estimated state feedback control. For more details, the reader can refer to the works on the topic: [9] [22], [7], [6], [8], [13], [5], [17].

The main problem in the majority of works, using decentralized observers to estimate state feedback control([19], [22],[15], [12]),..., is based on the fact that the nonlinear function of interconnection is uncertain and satisfies some conditions. This add more restrictive conditions on the synthesis of the gains of observation / control.

The basic idea of this work is to provide a non restrictive sufficient condition on nonlinear interconnection function which allow expressing stability conditions in terms of LMIs using the differential mean value theorem (DMVT)[21]. This sufficient condition enables to design a decentralized output feedback controller and to estimate the state of individual subsystems that can be used in the synthesis of the estimated state feedback control. Stability of the estimation error is analyzed using the convexity principle and the Lyapunov stability theory with an optimization of quadratic cost performance. The observer/control gains guaranteeing the global convergence of the proposed scheme is computed by LMI that allow large values of Lipschitz constants. The idea behind the DMVT is to assure $\partial V / \partial t+J<0$ for the well known and widely used Lyapunov function $V(x)=x^{T} P x$ (where $J$ is quadratic cost performance). The outcome is to ensure asymptotic convergence for a large scale interconnected systems.

This work is organized as follows. In Section 2, the problem formulation and some limitations of existing works are introduced. Next, the method of synthesis of the observer/control gain will be 
detailed. This method consists in LMIs feasibility conditions. The last section is devoted to the well know the performance of the proposed approach through a numerical example with a comparison to the work of [15], [10], [4] and [16].

Notations: The following notations will be used throughout this paper .

$-A^{T}$ represents the transposed matrix of $A$;

-for a square matrix $S, S>0(S<0)$ means that this matrix is positive definite (negative definite);

- the set $C o(x, y)=\{\lambda x+(1-\lambda) y, 0 \leq \lambda \leq 1\}$ is the convex hull of $x, y$;

$-e_{s}(j)=(\underbrace{0, \ldots, 0, \overbrace{1}^{j^{t h}}, 0, \ldots, 0}_{s-\text { components }})^{T} \in \mathbb{R}^{s}, s \geq 1$, is the vector of the canonical basis of $\mathbb{R}^{s}$.

\section{PROBLEM STATEMENT}

Consider a nonlinear interconnected system $\mathbf{S}$ composed of a finite number $\mathbf{N}$ of subsystems represented by:

$$
S_{i}: \dot{x}_{i}=A_{i} x_{i}+B_{i} u_{i}+h_{i}(t, x), y_{i}=C_{i} x_{i}
$$

where $x_{i} \in \mathbb{R}^{n_{i}}, u_{i} \in \mathbb{R}^{m_{i}}$ and $y(t) \in \mathbb{R}^{p_{i}}$ are respectively the subsystem state, input and output vectors. $A_{i}, B_{i}$ and $C_{i}$ are constant matrices of adequate dimensions. $h_{i}(t, x)$ : reflects the interconnection term illustrating the nonlinearity of $i^{\text {th }}$ subsystem.

The entire interconnected system $\mathbf{S}$ can be represented in a compact form as:

$$
S: \dot{x}=A x+B u+h(t, x), y=C x
$$

where $x^{T}=\left(x_{1}^{T}, \ldots, x_{N}^{T}\right), u=\left(u_{1}^{T}, \ldots, u_{N}^{T}\right)$ and $y=$ $\left(y_{1}^{T}, \ldots, y_{N}^{T}\right)$ are the global state, input and output vectors, respectively $\left(\sum_{i=1}^{N} n_{i}=n, \sum_{i=1}^{N} m_{i}=m, \sum_{i=1}^{N} p_{i}=p\right)$. $A=\operatorname{diag}\left\{A_{1}, \ldots, A_{N}\right\} ; B \stackrel{i=1}{=} \operatorname{diag}\left\{B_{1}, \ldots, B_{N}\right\} ; C=$ $\operatorname{diag}\left\{C_{1}, \ldots, C_{N}\right\}$ and $h(t, x)=\left(h_{1}(t, x)^{T}, \ldots, h_{N}(t, x)^{T}\right)^{T}$ is the global nonlinear interconnection function. The pair $\left(A_{i}, B_{i}\right)$ and $\left(A_{i}, C_{i}\right)$ are assumed to be stabilizable and detectable, respectively. The majority of work, treating the field of control/state observer of nonlinear interconnected systems, are based on the fact that the function $h_{i}(t, x)$ is uncertain and satisfies:

- Quadratic inequalities ([19], [22], [12]) $h_{i}(t, x)^{T} h_{i}(t, x) \leq$ $\alpha_{i}^{2} x^{T} H_{i} H_{i}^{T} x$

- The function norm ([15]) $\left\|h_{i}(t, x)\right\| \leq \alpha_{i}\|x\|$

where $\alpha_{i}$ are interconnection bounds (to be maximized) and $H_{i}$ are bounding matrices. This hypothesis, add more restrictive conditions to synthesize the observation/control gains. In the next section, the idea is to show that the above general optimal stabilization problem (the synthesis of an observer/control gains) can also be formulated as an LMIs problem using the principle of DMVT.

\section{OBSERVER/CONTROL GAIN DESIGN}

\subsection{Synthesis of observer and control gains}

This section is dedicated to present some steps to the proposed approach. First, assume that the Jacobian matrix of $h_{i}$ satisfies the following condition [21]:

$$
a_{j k}^{i} \leq \frac{\partial h_{i_{j}}(t, x)}{\partial x_{k}} \leq b_{j k}^{i}
$$

where $a_{j k}^{i}=\min _{Z \in \mathbb{R}^{n}}\left(\frac{\partial h_{i_{j}}(t, x)}{\partial x_{k}}(Z)\right)$

and $b_{j k}^{i}=\max _{Z \in \mathbb{R}^{n}}\left(\frac{\partial h_{i_{j}}(t, x)}{\partial x_{k}}(Z)\right)$.

The inequality (3) implies that the differentiable function $h_{i}$ is $\alpha_{i}$ Lipschitz where:

$$
\alpha_{i}=\sqrt{\sum_{j=1}^{j=q} \sum_{j=1}^{k=n} \max \left(\left|a_{j k}\right|^{2},\left|b_{j k}\right|^{2}\right)}
$$

The proposed decentralized observer of the overall system (2), composed from $N$ local observers, is given by :

$$
\left\{\begin{array}{c}
\dot{\hat{x}}=A \hat{x}+B u+L(y-\hat{y}) \\
\hat{y}=C \hat{x}
\end{array}\right.
$$

where $\hat{x}$ is the estimated state of the overall system and $L$ the observation gain matrix $\left(L=\operatorname{diag}\left\{L_{i}\right\}\right)$. Let's consider $\varepsilon=x-\hat{x}$ the estimation error. Then from the observer $[5]$ and the system (2) the dynamic of the global estimation error is described by:

$$
\dot{\varepsilon}=(A-L C) \varepsilon+h(t, x)
$$

Remark 1 : At this stage the nonlinear function $h$ is not considered in the synthesis of the observer gain. The knowledge of the non-linear interconnections is not required for the proposed solution. The observer structure of the global interconnected nonlinear system is totally decentralized.

Now, with the same reasoning used in [15], the local control law of each subsystem is given by:

$$
u_{i}=-K_{i} \hat{x}_{i}
$$

where $K_{i} \in \mathbb{R}^{m_{i} \times n_{i}}$ is the control gain matrix of the $i^{t h}$ subsystem. The control law of the global system (2) is expressed as

$$
u=-K \hat{x}
$$

where $K=\operatorname{diag}\left\{K_{i}\right\}$ is the block diagonal control gain matrix. The development of the global interconnected nonlinear system, using the control law $[8$, leads to

$$
\left\{\begin{array}{c}
\dot{x}=(A-B K) x+B K \varepsilon+h(t, x) \\
y=C x
\end{array}\right.
$$

Therefore, the augmented system including the overall system (9) and the global dynamics observation error system (6) is given by a state representation as:

$$
\underbrace{\left[\begin{array}{c}
\dot{x} \\
\dot{\varepsilon}
\end{array}\right]}_{\tilde{\tilde{x}}}=\underbrace{\left[\begin{array}{cc}
A-B K & B K \\
0 & A-L C
\end{array}\right]}_{\tilde{A}} \cdot \underbrace{\left[\begin{array}{c}
x \\
\varepsilon
\end{array}\right]}_{\tilde{x}}+\underbrace{\left[\begin{array}{c}
I_{n} \\
I_{n}
\end{array}\right] h(t, x)}_{\tilde{h}(t, \tilde{x})}
$$

The resulting system can be written by the state equations

$$
\left\{\begin{array}{c}
\dot{\tilde{x}}=\tilde{A} \tilde{x}+\Psi h(t, x) \\
x=\mathcal{L} \tilde{x}
\end{array}\right.
$$

where $\mathcal{L}=\left[\begin{array}{ll}I_{n} & 0\end{array}\right]$ and $\Psi=\left[\begin{array}{c}I_{n} \\ I_{n}\end{array}\right]$.

The problem is to find a way to obtain the control gain $K$ and the observation gain $L$ which can achieve the stability of the overall system. Moreover, the guaranteed cost control of the closed loop system is ensured. 


\subsection{Stability Analysis}

This section deals with the stability analysis and the decentralized guaranteed cost control of the closed loop system. To ensure, the following criteria (quadratic cost performance) is optimized:

$$
J=\int_{0}^{\infty}\left(x^{T} Q x+u^{T} R u\right) d t
$$

where $Q=Q^{T}>0$ and $R=R^{T}>0$ are given constant weighting matrices.Then, using the dynamic output feedback control $u=-K \hat{x}$, then the cost function 12 can be rewritten as follows:

$$
\tilde{J}=\int_{0}^{\infty}(\tilde{x}^{T} \underbrace{\left[\begin{array}{cc}
Q+K^{T} R K & -K^{T} R K \\
-K^{T} R K & K^{T} R K
\end{array}\right]}_{\tilde{Q}} \tilde{x}) d t
$$

The decentralized control law based on decentralized state observer is said to be a quadratic guaranteed cost control with cost matrix $P>0$ for the augmented system $(10)$ and the cost function $(13)$ if the closed loop system is quadratically stable [18]. The closed loop value of the cost function 13 satisfies the bound $J<\tilde{J}$ for all admissible nonlinearities.

Initially, the candidate Lyapunov function $V(\tilde{x})$ is defined by:

$$
V(\tilde{x})=\tilde{x}^{T} P \tilde{x}
$$

where Lyapunov matrix $P$ is defined by : $P=\left[\begin{array}{cc}P_{c} & 0 \\ 0 & P_{0}\end{array}\right]$. where $P_{c}=P_{c}^{T}=\operatorname{diag}\left\{P_{c i}\right\}$ and $P_{0}=P_{0}^{T}=\operatorname{diag}\left\{P_{0 i}\right\}$ are Lyapunov positive definite symmetric matrices. The aim, in what follows, is to determine conditions for which

$$
\frac{d}{d t} V(\tilde{x})+\tilde{x}^{T} \tilde{Q} \tilde{x}<0
$$

Noting $\tilde{h}(t, \tilde{x})$ by $\tilde{h}$. From 14 and according to 15 , we have:

$$
(\tilde{A} \tilde{x}+\tilde{h})^{T} P \tilde{x}+\tilde{x}^{T} P(\tilde{A} \tilde{x}+\tilde{h})+\tilde{x}^{T} \tilde{Q} \tilde{x}<0
$$

The equation 16) can be rewritten as:

$$
\tilde{x}^{T}\left(\tilde{A}^{T} P+P \tilde{A}\right) \tilde{x}+\tilde{x}^{T} \tilde{Q} \tilde{x}+\tilde{h}^{T} P \tilde{x}+\tilde{x}^{T} P \tilde{h}<0
$$

Proposition 1 Define the set $\mathcal{M}_{q, n}^{N}$ as follows:

$$
\begin{aligned}
\mathcal{M}_{q, n}^{N}= & \left\{v=\left(v_{11}^{1}, \ldots, v_{1 n}^{1}, \ldots, v_{q n}^{1}, \ldots, v_{q n}^{N}\right)\right. \\
& : a_{j k}^{i} \leq v_{j k}^{i} \leq b_{j k}^{i}, i=1, \ldots, N ; j=1, \ldots, q ; \\
& k=1, \ldots, n,\}
\end{aligned}
$$

The set $\mathcal{M}_{q, n}^{N}$ is a bounded convex domain of which the set of vertices is defined by:

$$
\begin{aligned}
\mathcal{V}_{\mathcal{M}_{q, n}^{N}}= & \left\{\gamma=\left(\gamma_{11}^{1}, \ldots, \gamma_{1 n}^{1}, \ldots, \gamma_{q n}^{1}, \ldots \gamma_{q n}^{N}\right):\right. \\
& \left.\gamma_{i j}^{i} \in\left\{a_{i j}^{i}, b_{i j}^{i}\right\}\right\}
\end{aligned}
$$

Proposition 2 (The DMVT for vector valued function [20]). Let $\Phi: \mathbb{R}^{n} \rightarrow \mathbb{R}^{q}$. Let $a, b \in \mathbb{R}^{n}$. $\Phi$ is assumed to be differentiable on $C o(a, b)$. Then, there are constant vectors $z_{1}, \ldots, z_{q} \in C o(a, b)$, $z_{i} \neq a, z_{i} \neq b$ for $i=1, \ldots, q$ such that

$$
\Phi(a)-\Phi(b)=\left(\sum_{i, j=1}^{q, n} e_{q}(i) e_{n}^{T}(j) \frac{\partial \Phi_{i}}{\partial x_{j}}\left(z_{i}\right)\right)(a-b) .
$$

In analogy to the approach of [1] [21] [20], and by applying Proposition 2 on the function $h_{i}$, there exist $z_{j} \in C o(x, 0)$, for all $j=1 \ldots q$, such that:

$$
h_{i}(t, x)-h_{i}(t, 0)=\left(\sum_{j, k=1}^{q, n} e_{q}(j) e_{n}^{T}(k) \frac{\partial h_{i_{j}}}{\partial x_{k}}\left(z_{j}\right)\right)(x-0)
$$

Assuming that $h_{i}(t, 0)=0$, then using Proposition 1 and 21 :

$$
h_{i}(t, x)=\Xi_{z_{i}} x
$$

where

$$
\Xi_{z_{i}}==\left[\sum_{j, k=1}^{q, n} e_{q}(j) e_{n}^{T}(k) \frac{\partial h_{i_{j}}}{\partial x_{k}}\left(z_{j}\right)\right]
$$

The vector $h(t, x)$ can be written as

$$
h(t, x)=\Xi_{z} x
$$

where $\Xi_{z}=\left[\Xi_{z_{1}}^{T}, \ldots, \Xi_{z_{N}}^{T}\right]^{T}$

Remark 2 : from equation [17, [15] [22] and [12] consider a transformation of the $\tilde{h}^{T} P \tilde{x}+\tilde{x}^{T} P \tilde{h}$ to express it in the form of Quadratic inequalities given in Section 21 (which remains conservative). To cope with this restriction, and using equation (11,

$$
\tilde{h}=\Gamma \tilde{x}
$$

where $\Gamma=\Psi \Xi_{z} \mathcal{L}$

Then, the condition for the asymptotic stability (using the assumption of [2]) with a guaranteed level of performance is given by:

$$
\tilde{x}^{T}\left(\tilde{A}^{T} P+P \tilde{A}+\tilde{Q}+\Gamma^{T} P+P \Gamma\right) \tilde{x}<0
$$

Theorem 1. The global system is stable in the sense of Lyapunov and the cost performance $(12)$ is guaranteed if there exist matrices $P=P^{T}, L=\operatorname{diag}\left(L_{1}, \ldots, L_{N}\right)$ and $K=\operatorname{diag}\left(K_{1}, \ldots, K_{N}\right)$ of appropriate dimensions such that the following LMI is feasible:

$$
\begin{aligned}
& \operatorname{Diag}\left(F\left(\gamma^{1}\right), \ldots, F\left(\gamma^{2^{N q n}}\right)\right)<0, \\
& \gamma^{i} \in \mathcal{V}_{\mathcal{M}_{q, n}^{N}} \text { for } i=1, \ldots, 2^{N q n} \\
& \text { where } \\
& F\left(\gamma^{i}\right)=\tilde{A}^{T} P+P \tilde{A}+\tilde{Q}+\Gamma^{T}\left(\gamma^{i}\right) P+P \Gamma\left(\gamma^{i}\right)
\end{aligned}
$$

To ensure it $\left(F\left(\gamma^{i}\right)<0\right)$, 27) can be transformed into LMIs, which can be solved in a computationally efficient manner by using the LMI optimization technique. The development of 27] leads to:

$$
\left(\begin{array}{ll}
X_{11} & X_{12} \\
X_{21} & X_{22}
\end{array}\right)<0
$$

where:

$$
\left\{\begin{array}{l}
X_{11}=A^{T} P_{c}+P_{c} A-K^{T} B^{T} P_{c}-P_{c} B K+\Xi_{z}^{T} P_{c}+P_{c} \Xi_{z} \\
\quad+Q+K^{T} R K \\
X_{12}=P_{c} B K-K^{T} R K+\Xi_{z}^{T} P_{0} \\
X_{21}=X_{12}^{T}=K^{T} B^{T} P_{c}-K^{T} R K+P_{0} \Xi_{z} \\
X_{22}=A^{T} P_{0}+P_{0} A-C^{T} L^{T} P_{0}+P_{0} L C+K^{T} R K
\end{array}\right.
$$

Notice that there are no effective algorithms for solving simultaneously the control problem and the observer one. Thus, to solve, we proceed in two steps (solve the control parameters first and then solve the observer parameters [15]). The first-step consists in multiplying the left-hand side and the right-hand side of 28 by:

$$
\left(\begin{array}{cc}
W & 0 \\
0 & I
\end{array}\right), W=W^{T}=P_{c}^{-1}>0
$$


The equation 28, becomes:

$$
\left(\begin{array}{cc}
Y_{11} & Y_{12}+W \Xi_{z}^{T} P_{0} \\
Y_{21}+P_{0} \Xi_{z} W & Y_{22}
\end{array}\right)<0
$$

Then, using the notations $Y=K W$ and $Z=P_{0} L$

$$
\left\{\begin{aligned}
Y_{11} & =W A^{T}+A W-Y^{T} B^{T}-B Y+W \Xi_{z}^{T}+\Xi_{z} W+W Q W \\
& \quad+Y^{T} R Y \\
Y_{12} & =B K-Y^{T} R K \\
Y_{21} & =Y_{12}^{T} \\
Y_{22} & =A^{T} P_{0}+P_{0} A-C^{T} Z^{T}+Z C+K^{T} R K
\end{aligned}\right.
$$

Unsing repeatedly the Schur complement formula, 30 can be transformed into the following inequality:

$$
\left[\begin{array}{cccc}
Y_{11} & Y_{12} & W E_{Z}^{T} & 0 \\
Y_{21} & Y_{22} & 0 & P_{0} \\
0 & P_{0} & -I & 0 \\
E_{Z} W & 0 & 0 & -I
\end{array}\right]<0
$$

Now, the determination of the control parameters (designed by the matrices $W$ and $Y$ ) becomes from the resolution of the matrix inequality $Y_{11}<0$. Using the Schur complement formula, the inequality $\left(Y_{11}<0\right)$ can be written as:

$$
\left(\begin{array}{ccc}
W A^{T}+A W-Y^{T} B^{T}-B Y+W \Xi_{z}^{T}+\Xi_{z} W & W & Y^{T} \\
W & -Q^{-1} & 0 \\
Y & 0 & -R^{-1}
\end{array}\right)<0
$$

Thereafter, the control gain matrix is given by:

$$
K=Y W^{-1}
$$

The second step is devoted to find the observation gain $L$.

Then, the determination of $P_{0}$ and $Z$ is given by substituting the parameters obtained from the first step and solving the LMI 31. The observation gain is given by:

$$
L=P_{0}^{-1} Z
$$

\section{APPLICATION OF THE PROPOSED APPROACH TO A MULTI-MACHINE POWER SYSTEM}

In this section, the efficiency of the proposed distributed dynamic output feedback controller through a numerical example is shown. Three machines power systems given in [16], [15] and [11] (shown in Fig. 1 are given, where generators 2 and 3 are assumed to have the same dynamics and the same parameters [3].

The following sections present the non-linear model used in this paper and the simulation results.

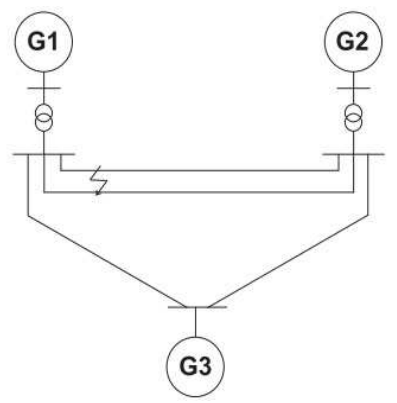

Fig. 1: Three-machine power system

\subsection{Power system nonlinear model}

The state vector of the $i^{\text {th }}$ subsystem is defined by (for system $S_{i}$ given in 11 :

$$
x_{i}(t)^{T}=\left[\begin{array}{llll}
\Delta \delta_{i}(t) & w_{i}(t) & \Delta P_{m i}(t) & \Delta X_{e i}(t)
\end{array}\right]
$$

where

$-\Delta \delta_{i}(t)=\delta_{i}(t)-\delta_{i 0}$;

$-\Delta P_{m i}(t)=P_{m i}(t)-P_{m i 0}$;

$-\Delta X_{e i}(t)=X_{e i}(t)-X_{e i 0}$;

$-u_{i}(t)$ is the control vector of the $i^{t h}$ subsystem, $u_{i}(t)=$ $\Delta X_{e i}(t)$;

$-y_{i}(t)$ is the output vector of the $i^{t h}$ subsystem, $y_{i}(t)=\Delta \delta_{i}(t)$;

$-\delta_{i}(t)$ is the rotor angle for the $i^{t h}$ machine, in radian;

$-w_{i}(t)$ is the relative speed for the $i^{t h}$ machine, in radian;

$-P_{m i}(t)$ is the mechanical power for the $i^{t h}$ machine, in pu;

$-X_{e i}(t)$ is the steam valve for the $i^{t h}$ machine, in pu;

$-h_{i}(t, x(t))=\sum_{j=1, j \neq i}^{N} p_{i j} G_{i j} g_{i j}\left(x_{i}, x_{j}\right)$ is a nonlinear function vector characterizing the interconnection between subsystems;

$-\delta_{i 0}, P_{m i 0}, X_{e i 0}$ are the nominal values of $\delta_{i}(t), P_{m i}(t)$ and $X_{e i}(t)$.

$A_{i}=\left[\begin{array}{cccc}0 & 1 & 0 & 0 \\ 0 & \frac{-D_{c i}}{2 H_{i}} & \frac{-w_{0}}{2 H_{i}} & 0 \\ 0 & 0 & \frac{-1}{T_{m i}} & \frac{K_{m i}}{T_{m i}} \\ 0 & \frac{-K_{e i}}{T_{e i} R_{i} w_{0}} & 0 & \frac{-1}{T_{e i}}\end{array}\right] \quad B_{i}=\left[\begin{array}{c}0 \\ 0 \\ 0 \\ \frac{1}{T_{e i}}\end{array}\right]$

$C_{i}^{T}=\left[\begin{array}{l}1 \\ 0 \\ 0 \\ 0\end{array}\right] \quad G_{i j}=\left[\begin{array}{c}0 \\ \frac{w_{0} E_{q i}^{\prime} E_{q j}^{\prime} B_{i j}}{2 H_{i}} \\ 0 \\ 0\end{array}\right]$

and $g_{i j}\left(x_{i}, x_{j}\right)=\sin \left(\delta_{i}(t)-\delta_{j}(t)\right)-\sin \left(\delta_{i 0}-\delta_{j 0}\right)$. Where:

$p_{i j} \quad$ Constant of either 1 or $0\left(P_{i j}=0\right.$ means that $j^{\text {th }}$ machine has no connection with $i^{\text {th }}$ machine;

$H_{i} \quad$ Inertia constant for the $i^{\text {th }}$ machine, in second;

$D_{c i} \quad$ Damping coefficient for the $i^{t h}$ machine, in pu;

$T_{m i} \quad$ Time constant for $i^{\text {th }}$ machine's turbine, in second;

$K_{m i} \quad$ Gain of $i^{t h}$ machine's turbine;

$X_{d_{i}} \quad$ the direct axis reactance of the $i^{\text {th }}$ machine, in p.u.

$X_{d_{i}}^{i} \quad$ the direct axis transient reactance of the $i^{t h}$ machine, in p.u.

$X_{T_{i}} \quad$ the transformer reactance of the $i^{\text {th }}$ machine, in p.u.

$T_{e i} \quad$ Time constant of the $i^{t h}$ machine's speed governor, in second;

$K_{e i} \quad$ Gain of the $i^{t h}$ machine's speed governor;

$R_{i} \quad$ Regulation constant of the $i^{t h}$ machine, in $\mathrm{pu}$;

$B_{i j} \quad$ Nodal susceptance between $i^{t h}$ and $j^{t h}$ machines, in pu;

$w_{0} \quad$ Synchronous machine speed, $w_{0}=2 \pi f_{0}$, in radian $/ \mathrm{s}$;

$E_{q i}^{\prime} \quad$ Internal transient voltage for $i^{t h}$ machine, in pu, assumed to be constant;

$E_{q j}^{\prime} \quad$ Internal transient voltage for $j^{t h}$ machine, in pu, assumed to be constant; 


\subsection{Simulation Results}

The parameters of the three interconnected power systems [16] are summarized in table I:

\begin{tabular}{|c|c|c|c|}
\hline & Generator1 & Generator2 & Generator3 \\
\hline$X_{d}(\mathrm{pu})$ & 1.863 & 2.36 & 2.36 \\
\hline$X_{d}^{\prime}(\mathrm{pu})$ & 0.257 & 0.319 & 0.319 \\
\hline$X_{T}(\mathrm{pu})$ & 0.129 & 0.11 & 0.11 \\
\hline$X_{a d}(\mathrm{pu})$ & 1.712 & 0.712 & 0.712 \\
\hline$T_{d 0}^{\prime}(\mathrm{pu})$ & 6.9 & 7.96 & 7.96 \\
\hline$H(s)$ & 4 & 5.1 & 5.1 \\
\hline$D_{c}(\mathrm{pu})$ & 5 & 3 & 3 \\
\hline$T_{m}(\mathrm{~s})$ & 0.35 & 0.35 & 0.35 \\
\hline$T_{e}(\mathrm{~s})$ & 0.1 & 0.1 & 0.1 \\
\hline$R$ & 0.05 & 0.05 & 0.05 \\
\hline$K_{m}$ & 1 & 1 & 1 \\
\hline$K_{e}$ & 1 & 1 & 1 \\
\hline$w_{0}(\mathrm{rad} / \mathrm{s})$ & 314.159 & 314.159 & 314.159 \\
\hline \multicolumn{4}{|r}{} \\
\end{tabular}

Table 1. : Parameters of the three interconnected power systems

According to the model, the studied power system can be described by the following state equations:

$$
\left\{\begin{aligned}
\dot{x}_{1}(t)= & A_{1} x_{1}(t)+B_{1} u_{1}(t)+G_{12} g_{12}\left(x_{1}(t), x_{2}(t)\right) \\
& +G_{13} g_{13}\left(x_{1}(t), x_{3}(t)\right) \\
\dot{x}_{2}(t)= & A_{2} x_{2}(t)+B_{2} u_{2}(t)+G_{21} g_{21}\left(x_{2}(t), x_{1}(t)\right) \\
& +G_{23} g_{23}\left(x_{2}(t), x_{3}(t)\right) \\
\dot{x}_{3}(t)= & A_{3} x_{3}(t)+B_{3} u_{3}(t)+G_{31} g_{31}\left(x_{3}(t), x_{1}(t)\right) \\
& +G_{32} g_{32}\left(x_{3}(t), x_{2}(t)\right)
\end{aligned}\right.
$$

where $G_{i j}=\left[\begin{array}{llll}0 & \alpha_{i j} & 0 & 0\end{array}\right]^{T}$ are given by [4]- [16]: $\alpha_{12}=\alpha_{13}=-27.49$

$\alpha_{21}=\alpha_{23}=\alpha_{31}=\alpha_{32}=-23.10$

with $\alpha_{i j}$, represents the midpoints of $\frac{w_{0} E_{q i}^{\prime} E_{q j}^{\prime} B_{i j}}{2 H_{i}}$. The nonlinear interconnection functions of the three interconnected machines can be expressed as : $h_{1}(x)=\left[\begin{array}{c}0 \\ h_{12} \\ 0 \\ 0\end{array}\right] ; h_{2}(x)=\left[\begin{array}{c}0 \\ h_{22} \\ 0 \\ 0\end{array}\right] ; h_{3}(x)=\left[\begin{array}{c}0 \\ h_{32} \\ 0 \\ 0\end{array}\right]$

with

$$
\left\{\begin{array}{l}
h_{12}=\alpha_{12} g_{12}\left(x_{1}, x_{2}\right)+\alpha_{13} g_{13}\left(x_{1}, x_{3}\right) \\
h_{22}=\alpha_{21} g_{21}\left(x_{2}, x_{1}\right)+\alpha_{23} g_{23}\left(x_{2}, x_{3}\right) \\
h_{32}=\alpha_{31} g_{31}\left(x_{3}, x_{1}\right)+\alpha_{32} g_{32}\left(x_{3}, x_{2}\right)
\end{array}\right.
$$

After choosing the weighting matrices of the cost function as $Q=10^{-2} I_{12} R=10^{-3} I_{3}$, the control and observer gain matrix found from the resolution of the LMIs problem $32,33.34$ are $K=\operatorname{diag}\left\{K_{i}\right\}, i=1,2,3$ and $L=\operatorname{diag}\left\{L_{i}\right\}, i=1,2,3$ such that:

$$
\begin{aligned}
K_{1} & =\left[\begin{array}{llll}
462.321 & 81.115 & 229.122 & 19.084
\end{array}\right] \\
K_{2} & =\left[\begin{array}{llll}
459.784 & 81.491 & 211.049 & 18.536
\end{array}\right] \\
K_{3} & =\left[\begin{array}{llll}
459.784 & 81.491 & 211.049 & 18.536
\end{array}\right]
\end{aligned}
$$

$$
\begin{aligned}
L_{1} & =\left[\begin{array}{c}
99.1663 \\
97.3051 \\
1.5329 \\
-7.6471
\end{array}\right] ; L_{2}=\left[\begin{array}{c}
88.2933 \\
75.1120 \\
1.3593 \\
-6.7894
\end{array}\right] ; \\
L_{3} & =\left[\begin{array}{c}
88.2933 \\
75.1120 \\
1.3593 \\
-6.7894
\end{array}\right]
\end{aligned}
$$

First, in Fig. 2 the observer error is given (for the rotor angle of the three subsystems $e=x_{1 i}-\hat{x}_{1 i}$ ).

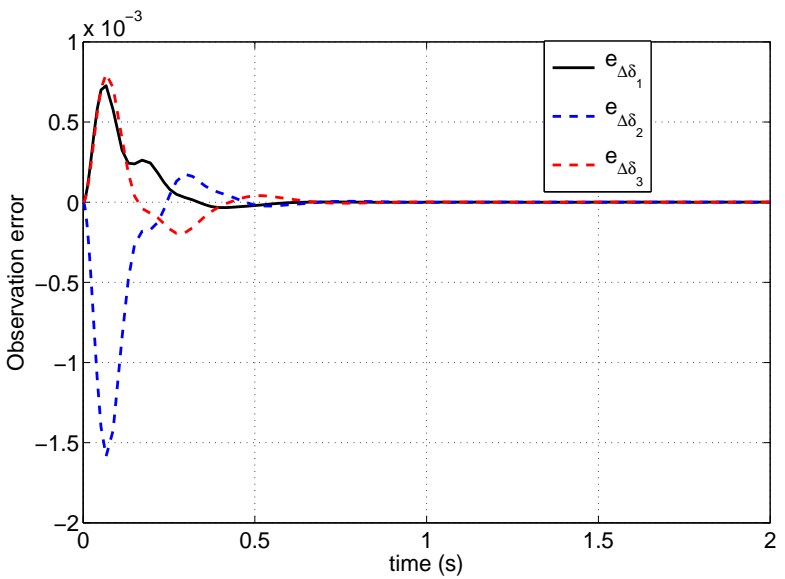

Fig. 2: Evolution of the estimation error

Fig 2 shows that the estimation error converges to zero with a very small variation (0.005). The behavior of the $\|x-\hat{x}\|$ is given in Fig 3 It is clearly that the stability and convergence of the proposed method are ensured.

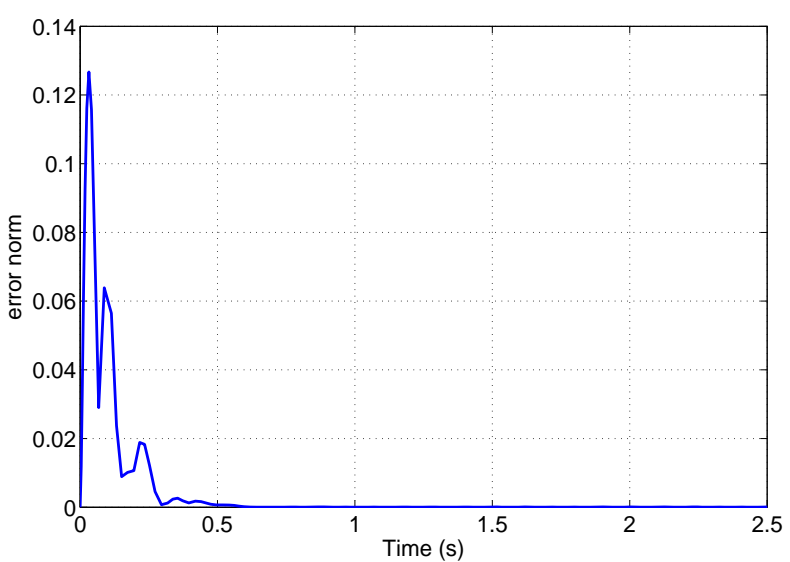

Fig. 3: Evolution of $\|x-\hat{x}\|$

Secondly, in Fig. 4 the evolution of the estimated rotor angle $\hat{x}_{1}$ 
is presentend and compared with the results of [15]. Fig 4 shows clearly the contribution added by the method proposed in this paper. Indeed, this method reduces the peaks evolution (during transitional regime) and the convergence time which is defined as the time when the value of the state reached a range of $\pm 5 \%$ of the origin.

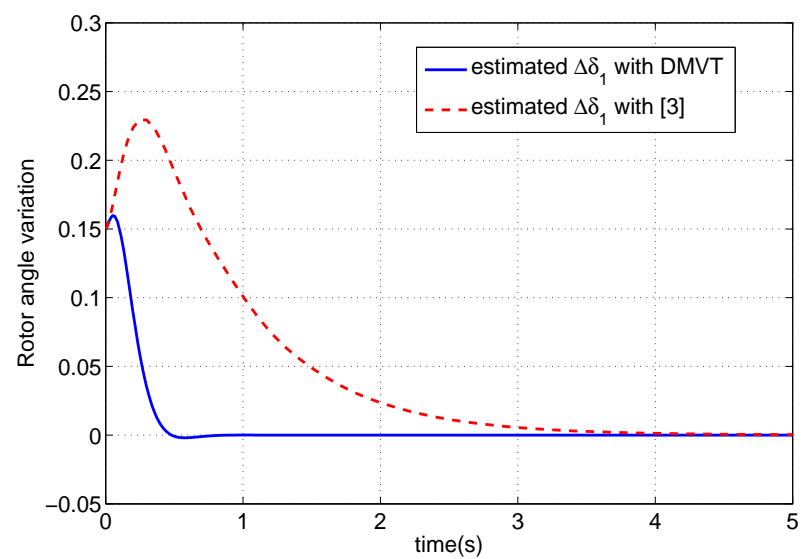

Fig. 4: Evolution of the estimated rotor angle $\hat{x}_{1}$

Concerning the last point, and in order to prove the contribution acquired on the convergence time (Table II), the results are compared with other methods ([16]-[10]-[4]-[15]).

\begin{tabular}{|c|c|}
\hline & Convergence Time (s) \\
\hline Proposed method & 0.3 \\
\hline Wang[16] & 1.6 \\
\hline Siljack[10] & 1.6 \\
\hline Guo[4] & 1.8 \\
\hline Tlili[15 & 2.4 \\
\hline
\end{tabular}

Table 2. : Convergence Time

It is demonstrated from Table II that the proposed result ensures convergence at the least time compared to other methods.

Remark 3 :

The proposed method provides solutions even for huge Lipschitz constants. In this work, the use of bounds $\alpha_{i}>10^{3}$ is tolerated by the system without losing stability, but in [15], a loss of stability is observed.

Remark 4 :

As it can be seen, the cost of more demanding solve LMI is high. However, the use of the DMVT approach gives a less restrictive LMI synthesis condition. For the proposed method (and all LMIbased approaches), the constant observer gain is computed off line. Thus, it is suitable to real-time application.

Remark 5 :

In [4] and [10], the local control law of each subsystem needs the knowledge of the state, while the proposed local control law in this work is based on the state estimation. The decentralized observers is designed to estimate the state of individual subsystem which are used for the feedback controller.

\section{CONCLUSIONS}

An efficient decentralized controller and observer for a class of large-scale interconnected nonlinear systems is presented. The use of the DMVT had ensured that the stability analysis is performed with non restrictive sufficient condition to ensure asymptotic convergence.

The developed method is then applied to stabilize power systems with three interconnected machines. Numerical results have confirmed the high performances of estimation and control offered by the proposed DMVT design method. The remaining open questions are the experimental test of the proposed method and its application to large scale power systems.

\section{REFERENCES}

[1] M. Benallouch, M. Boutayeb, and M. Zasadzinski. Observers design for one-sided lipschitz discrete-time systems. Syst. Control Letters, 61:879-886, 2012.

[2] S. Boyd, L. El Ghaoui, E. Ferron, and V. Balakrishnan. Linear matrix inequalities in systems and control theory. Studies in Applied Mathematics SIAM, Philadelphia, 15 edition, 1994.

[3] S. Elloumi and E. B. Braiek. Robust decentralized control for multimachine power systemsthe lmi approach. In IEEE Proc. Conf. on Decision and Control, page vol. 6, IEEE International Conference on Systems, Man and Cybernetics, 2006.

[4] Y. Wang G. Guo and D. J. Hill. Nonlinear decentralized control of large-scale power systems. Autmatica, 36:1275-1289, 2000.

[5] D.W.C. Ho and G. Lu. Robust stabilization for a class of discrete-time nonlinear systems via output feedback: The unified lmi approach. Int. J. of Control, 76:105-115, 2003.

[6] $\mathrm{H}$. Hu and D. Zhao. Decentralized $\mathcal{H}_{\infty}$ control for uncertain interconnected systems of neutral type via dynamic output feedback. Abstract and Applied Analysis, 2014:1-11, 2014.

[7] C. Hua, L. Zhang, and X. Guan. Decentralized output feedback controller design for nonlinear interconnected systems with unknown control direction and time-varying delays. Int. J. of Adaptive Control and Signal Processing, 28:1160-1173, 2014.

[8] Y. Liu and X.Y. Li. Decentralized robust adaptive control of nonlinear systems with unmodeled dynamics. IEEE Trans. on Autom. Control, 47:848-856, 2002.

[9] X. Mao, W. Liu, L. Hu, Q. Luo, and J. Lu. Stabilization of hybrid stochastic differential equations by feedback control based on discrete-time state observations. Syst. Control Letters, 73:88-95, 2014.

[10] D.D. Siljak and D. Stipanovic. Robust stabilization of nonlinear systems. Math. Probl. Eng., 6:461-493, 2000.

[11] D.D. Siljak and A.I. Zecevic. Control of large-scale systems: Beyond decentralized feedback. Annual Rev. in Control, 29:169-179, 2005.

[12] S.S. Stankovic and D.D. Siljak. Robust stabilization of nonlinear interconnected systems by decentralized dynamic output feedback. Syst. Control Letters, 58:271-275, 2009.

[13] S.S. Stankovic, D.M. Stipanovic, and D.D. Siljak. Decentralized dynamic output feedback for robust stabilization of a class of nonlinear interconnected systems. Autmatica, 43:861-867, 2007. 
[14] A. Thabet, M. Boutayeb, and M.N. Abdelkrim. Real time dynamic state estimation for power system. Int J. of Computer Applications, 38-2:11-18, 2012.

[15] A.S. Tlili and N.B. Braiek. Decentralized observer based guaranteed cost control for nonlinear interconnected systems. Int J. of Control Automation, 2:29-45, 2009.

[16] Y. Wang, D.J. Hill, and G. Guo. Robust decentralized control for multimachine power systems. IEEE Trans. on Cir. and Syst., 45(3):271-279, 1998.

[17] X.G. Yan, C. Edwards, and S.K. Spurgeon. Decentralized robust sliding mode control for a class of nonlinear interconnected systems by static output feedback. Autmatica, 40:113120, 2004.

[18] G.H. Yang, J.L. Wang, and Y.C. Soh. Reliable guaranteed cost control for uncertain nonlinear systems. IEEE Trans. on Autom. Control, 45:2188-3192, 2000.

[19] A.I. Zecevic and D.D. Siljak. Estimating the region of attraction for large-scale systems with uncertainties. Autmatica, 46:445-451, 2010

[20] A. Zemouch and M. Boutayeb. A unified $\mathcal{H}_{\infty}$ adaptive observer synthesis method for a class of systems with both lipschitz and monotone nonlinearities. Syst. Control Letters, 58:282-288, 2009.

[21] A. Zemouch, M. Boutayeb, and G.I. Bara. Observers for a class of lipschitz systems with extension to $\mathcal{H}_{\infty}$ performance analysis. Syst. Control Letters, 57:18-27, 2008.

[22] Y. Zhu and P.R. Pagilla. Decentralized output feedback control of a class of large-scale interconnected systems. J. of Mathematical Control and Information, 24:57-69, 2007. 